\title{
Elasticidades de transmissão espacial de preço do milho no Brasil: um estudo para o Mato Grosso
}

\section{Elasticity of spatial price transmission of the corn price market in Brazil: a study for Mato Grosso}

\author{
Geraldo Costa Junior ${ }^{1}$ \\ Nataliya Dimitrova Popova² \\ Tarik Canaan Thomé Tanus ${ }^{3}$ \\ João Gomes Martines Filho ${ }^{4}$
}

\section{Resumo}

O milho se situa entre os cerais mais produzidos no mundo. Além disso, o ceral é um bem essencial para a alimentação humana e, em alguns países, é também uma importante fonte de energia renovável. No Brasil, o estado de Mato Grosso é um dos principais produtores do grão e vem expandindo a sua integração com o mercado externo. Neste contexto, o objetivo deste estudo é analisar o processo de transmissão de preços entre as áreas produtoras de milho no Mato Grosso e entre estas e o mercado externo. O período de análise é de janeiro de 2009 a abril de 2015. Para este efeito, o quadro teórico da Lei do Preço Único (LOP) foi utilizado e a metodologia baseou-se no teste de causalidade de Granger e no modelo VECM. Os resultados obtidos apontam para a existência de uma relação causal unidirecional no sentido de Paranaguá para os mercados de milho de Mato Grosso. Em relação aos testes de cointegração, apenas as regiões de Canarana, Lucas do Rio Verde e Sapezal apresentaram relacionamento de longo prazo com Paranaguá. Por outro lado, a comparação entre as áreas de produção em MT mostra que existe uma relação de cointegração entre todas as praças analisadas, indicando um mercado de milho integrado no estado.

Palavras-chave: Transmissão, milho, preços, cointegração, comercialização.

\begin{abstract}
The present study aims at analyzing corn, which, due to its leadership in the grain market in terms of global production and its versatile use, has increased its importance in terms of food security and as a source of renewable energy. In Brazil, the state of Mato Grosso, which, besides its productive representativeness, has expanded its integration with the external market of grains, stands out. In this context, the goal of this study is to analyze the price transmission process between the corn producing areas in Mato Grosso and the foreign market for the period January 2009 - April 2015. For this purpose, the theoretical framework of the Law of One Price (LOP) was used and the methodology of the Granger causality test and the vector error correction model (VECM) estimation was applied. The obtained results point towards the existence of a one-way causal relationship in the Granger sense from Paranaguá towards the corn markets of Mato Grosso. Regarding the cointegration tests, only the regions of Canarana, Lucas do Rio Verde and Sapezal were found to have a long-term relationship with Paranaguá. On the other hand, the comparison between the production areas in Mato Grosso shows that there is a cointegration relationship between them, indicating an integrated corn market.

1 Doutorando em Economia Aplicada - ESALQ/USP. E-mail: geraldocir@gmail.com

2 Doutoranda em Economia Aplicada - ESALQ/USP . E-mail : nataliadpopova@googlemail.com

${ }^{3}$ Graduando em Ciências Econômicas - ESALQ/USP. E-mail : tarik.thome@gmail.com

${ }^{4}$ Professor Titular do Departamento de Economia, Administração e Sociologia - ESALQ/USP. E-mail : martines@usp.br
\end{abstract}


Key-words: Transmission, corn, prices, cointegration, trade.

JEL: Q02; Q10; R19

Enviado em: 5/03/2016

Aprovado para publicação em: 10/08/2016

\section{INTRODUÇÃO}

O mercado do milho em grão e seus derivados é um dos segmentos mais importantes do agronegócio mundial. Segundo o United States Department of Agriculture (USDA), o milho é elencado como o cereal mais produzido no mundo, se consolidando internacionalmente entre as commodities mais comercializadas. O milho integra a base de diferentes segmentos e mercados, sendo de fundamental importância para a nutrição humana e animal, além de poder ser utilizado como fonte de energia, como para a produção de etanol.

De acordo com o USDA, a produção mundial de milho na safra 2014/15, última safra completa, foi de 1.008,52 milhões de toneladas, com alta de $2 \%$, em relação à safra 2013/14. Os maiores produtores são Estados Unidos, China e Brasil, correspondendo à $35,8 \%, 21,4 \%$ e $8,4 \%$ em relação a produção mundial, respectivamente (USDA, 2015). De acordo com dados da Companhia Nacional de Abastecimento (CONAB), o Brasil deve finalizar a safra 2014/15 com uma produção de 85 milhões de toneladas, alta de 6,3\% em relação à safra anterior (CONAB, 2015). Entre o período 1970-2010, a produção brasileira cresceu significativamente, como resultado principalmente do aumento na produtividade e área plantada. Entretanto, a produção brasileira representa apenas $8 \%$ da produção mundial desse produto no período analisado (CRUZ JR. et al., 2016).

No que se refere às exportações de milho, os principais players do mercado são Estados Unidos, Brasil e Ucrânia, registrando 34,4\%, 23,6\% e 16,8\% em relação ao total exportado de 137,79 milhões de toneladas, em 2015, respectivamente (USDA, 2015). Uma característica do mercado internacional de milho é a transitoriedade em relação aos principais produtores e exportadores, de modo que não necessariamente os principais produtores serão, também, os principais exportadores. Nesse sentido, o mercado internacional de milho é tido como um dos mais dinâmicos mercados de commodities.

Tradicionalmente, a demanda por milho tem sido determinada pela alimentação humana e nutrição animal. Segundo a Secretaria do Comércio Exterior, do Ministério de Indústria e Comércio (SECEX/MDIC) o consumo mundial de milho é de 944,3 milhões de toneladas. Estados Unidos, China e União Europeia abrangem mais de $62 \%$ da demanda mundial do milho, com $31,4 \%, 22,45 \%$ e $8 \%$, respectivamente (SECEX, 2015). O Brasil se tornou um dos maiores consumidores de milho nos últimos anos e foi responsável por $6 \%$ do consumo mundial na safra 2013/14, segundo USDA.

O milho tem sido também utilizado para a produção de etanol, modalidade mais comum nos Estados Unidos e ainda pouco utilizada no Brasil. Segundo dados da União da Indústria de Cana-de-Açúcar (UNICA), até 2020 o Brasil necessitará de 100 novas usinas de etanol, em decorrência da produção de combustível a partir do milho. As próximas seções caracterizam a produção de milho no Brasil e no estado do Mato Grosso.

No contexto brasileiro, a cadeia produtiva de milho é uma das mais importantes do agronegócio nacional (TABOSA; IRFFI; PENNA, 2014). A produção brasileira dessa commodity é caracterizada tanto por lavouras comerciais altamente 
tecnificadas, como por lavouras de subsistências com baixo grau de tecnologia. A produção de milho se encontra em todos os estados do país. Até recentemente, a região Sul era o principal produtor, mas nos últimos anos os estados da região CentroOeste passaram a ser os mais relevantes nesse contexto, com Mato Grosso tornandose o estado com a maior área plantada de milho no país (WESTERICH FILHO, 2014). Esta região também se destacou como o maior exportador de milho no período recente.

O Brasil não é somente um grande produtor de milho, mas também um grande consumidor, o que significa que, além da exportação, uma parte significativa da produção desse produto no país é direcionada para o consumo interno. O uso do milho no Brasil é caracterizado por sua versatilidade. O grão é usado como matéria-prima em diferentes mercados e ao mesmo tempo tem o papel de insumo para diversos sistemas agroindustriais. Os setores de aves e suínos são responsáveis por mais de metade do consumo total do milho no país (WESTERICH FILHO, 2014).

A formação dos preços do milho no Brasil mudou a partir dos anos 1990, por causa da abertura da economia nacional (Sousa, Braga e Cunha, 2010). O preço doméstico do grão se tornou muito mais influenciado pelo mercado internacional, que levou a mudanças na comercialização dessa commodity e tornou o mercado interno de milho muito menos influenciado pelo governo (CHIODI, 2006). No entanto, ainda existem desafios para o setor brasileiro de milho, que devem ser enfrentadas para 0 produto se tornar mais competitivo no contexto internacional.

O estado do Mato Grosso está localizado na região Centro-Oeste do território brasileiro, seus estados vizinho são Amazonas, Pará, Tocantins, Goiás, Mato Grosso do Sul e Rondônia. Tal região é reconhecida pela produção agrícola, principalmente de milho e soja, destacando-se como um dos maiores produtores de grãos do Brasil. Segundo Moraes et al. (2015), o estado de Mato Grosso é o maior produtor agrícola do país atualmente e tal posição pode ser entendida como sendo resultado das condições favoráveis que o estado oferece como clima, topografia, grandes áreas para produção, modernização da agropecuária e investimento em tecnologia e genética.

A produção de milho do Mato Grosso representa cerca de 25,9\% da produção nacional, confirmando sua posição de maior produtor nacional de milho, segundo a Conab. Dados do Instituto Mato Grossense de Economia Agrícola (IMEA) apontam que o município de Sorriso é o maior produtor do cereal no país e, dentre os 10 municípios que mais produzem milho, seis se encontram no Mato Grosso (IMEA, 2015).

A produção de milho e soja no Mato Grosso é segmentada em duas safras durante o ano. A soja possui apenas uma safra, que se estende de novembro a abril, enquanto que o milho possui duas safras. A primeira safra do milho coincide temporalmente com a da soja e a segunda safra do milho, chamada de "safrinha", se estende de junho a setembro. No estado do Mato Grosso a produção de milho se concentra na segunda safra, que foi introduzida na região nos anos 2000 . O estado produziu 458,2 mil toneladas na primeira safra de 2014/15, 8,5\% acima da anterior, com produtividade de $7200 \mathrm{~kg} /$ há. Na segunda safra, a produção atingiu 20,305 milhões de toneladas, $15,2 \%$ acima da safra anterior, com produtividade de 6056 $\mathrm{kg} / \mathrm{ha}$ (CONAB, 2015).

O milho da primeira safra é principalmente direcionado para o mercado nacional, enquanto a colheita da segunda safra é usada para exportações. Tal fato implica a existência de sazonalidade nas exportações, que aumentam durante a safrinha de milho. Ademais, segundo Allen e Valdes (2016), o milho produzido na 
segunda safra se tornou um competidor do grão norte-americano no mercado internacional durante os meses de setembro a janeiro, que normalmente é abastecido pela produção dos países do hemisfério norte.

No Brasil, o estado de Mato Grosso é o principal exportador de milho, responsável por 50\% da exportação nacional em 2015, segundo SECEX (2015). Os principais países de destino desta produção foram Irã, Vietnã e Coreia do Sul. A demanda externa alcançou cerca de $68,37 \%$ do montante de milho produzido no estado, porém o maior obstáculo encontrado foi a dificuldade logística, o que dificulta a competitividade de preços frente a outros ofertantes do mercado.

Em relação a demanda interna do grão, cerca de $20,85 \%$ do montante produzido na última safra no estado foi destinado ao mercado interno, elevação de $8 \%$ frente a safra 2013/14. Esse aumento se deu pelo crescimento das indústrias de etanol a partir do processamento de milho, juntamente com a elevação de indústrias de nutrição animal, impulsionado pelo elevado preço da arroba do boi de modo ao incremento de confinamentos.

As propriedades de produção no estado de Mato Grosso são caracterizadas por grandes extensões se comparadas às propriedades de outras regiões no país. Embora o estado seja um grande produtor de milho, ele não é um consumidor relevante desse grão.

Quanto à comercialização dessa commodity, no estado predomina a utilização de contratos futuros, que permitem que a venda aconteça quando o milho ainda está na lavoura. A produção de primeira safra do milho do Mato Grosso tem como principais compradores cooperativas, fabricantes de rações e criadores de suínos e de aves principalmente situados nas regiões Sul e Sudeste (KAPPES, 2013). A utilização do mercado futuro faz que os preços na região não obedeçam às leis de oferta $e$ demanda no mercado, porque as expectativas no momento de venda afetam o preço do grão (Miranda e Garcia, 2012).

Além disso, os preços na região apresentam certa sazonalidade. Uma queda nos preços entre fevereiro e julho pode ser observada e um aumento no período entre abril e maio, que corresponde a época de pré-colheita na segunda safra (Mattos e Silveira, 2015). Na primeira safra os preços de milho são influenciados por fatores internos, enquanto os preços do milho da safrinha, que é direcionado para o mercado externo, são influenciados por condições internacionais (MATTOS; SILVEIRA, 2015).

Por fim, o Brasil se mostra um importante player no comércio internacional de milho, exportando, em 2015, 36\% da produção nacional. Desse montante exportado, $50 \%$ advém do estado do Mato Grosso, revelando a relevância de uma abordagem detalhada do estado, para o entendimento da comercialização externa do Brasil. Esses dados reforçam a importância de uma análise da integração de preços entre o mercado externo e interno, identificando uma relação mais assídua entre os mercados.

Dada a importância do Brasil no mercado internacional de milho e o peso do estado do Mato Grosso nas exportações do grão, o objetivo deste trabalho foi verificar a existência da transmissão de preços entre as principais praças produtoras do estado e o mercado externo. No caso de existência de transmissão, buscou-se ainda identificar seu sentido e magnitude. Este trabalho se diferencia dos demais por oferecer uma análise mais profunda e focada nas praças do estado do Mato Grosso e a ligação destas com o mercado externo. Os resultados contribuem no entendimento do processo de formação do preço interno do milho no estado e influências do resto do mundo sobre ele. Além disso, este estudo é uma continuação de outros estudos 
feitos anteriormente com foco na transmissão de preços entre diferentes estados (CHIODI, 2006; SOUSA; BRAGA; CUNHA, 2010; TABOSA; IRFFI; PENNA, 2014).

O conhecimento da transmissão dos preços dessa commodity é importante para orientação de todos os agentes inseridos na cadeia produtiva do milho. Desse modo, produtores e compradores de milho do estado do Mato Grosso, ao utilizar como referência o nível de transmissão entre os preços, podem ajustar-se para otimizar seu processo de tomada de decisão e melhor comercializar seu produto.

\section{REVISÃO DE LITERATURA}

\section{Transmissão de preços}

A crescente abertura das economias nacionais potencializa a possibilidade de ocorrência de transmissão de preços entre diferentes mercados, sejam estes mercados financeiros, de commodities, mercados cambiais, entre outros.

Segundo Saadi (2011), a transmissão de preços entre diferentes commodities é explicada pela relação de substituição e complementaridade entre estas. A transmissão de preços de mercados não agrícolas para agrícolas é sustentada pela estrutura de custos de produção e tecnologia, além de drivers do mercado financeiro que sustentam expectativas, fluxos especulativos e relações dos contratos futuros com o mercado spot. Por fim, a transmissão espacial de preços é sustentada por diretrizes de arbitragem e consequência da Lei do Preço Único.

Neste contexto, Stigler (2011) afirma que a transmissão de preços entre diferentes países que se enquadram em um mesmo mercado relevante pode ser influenciada por políticas de comercialização, acarretando forte influência na transmissão de preços.

No tocante à identificação e mensuração da transmissão de preços, Fackler e Goodwin (2001) iniciam uma abordagem básica, em vista de novos métodos econométricos, utilizando regressão simples e correlação de preços, auxiliando novos métodos para análise de transmissão de preços, como VECM e VAR. Listori e Esposti (2012), elencam a importância da utilização do VECM como uma ferramenta econométrica sofisticada para identificar transmissão de preços em nível nos mercados de produtos agrícolas.

Goodwin e Piggott (1999) utilizaram o modelo de VECM por regimes ao analisar a transmissão de preços diários entre o mercado central e três mercados locais de milho e soja na Carolina do Norte (USA). Greb et al. (2011), utilizou a ferramenta VECM para identificar transmissão de preço entre o mercado internacional dos principais cereais e o mercado doméstico da África.

Em relação à transmissão de preços entre os mercados internos e externos, Coronel et al. (2010) estuou a transmissão de preços de trigo entre a Argentina e o mercado externo. Os autores concluíram que as variações de preço internacional foram quase que completamente repassadas ao mercado argentino. Silva et al. (2005) analisaram a transmissão de preços da soja entre o Brasil e os Estados Unidos. Entre os resultados, verificou-se que o preço da soja spot no Brasil foi explicada em 32,97\% pelo preço da soja americana. Da mesma forma, Margarido e Sousa (1998) concluíram que o Brasil não é formador de preço da soja, mas sofre transferências de preços internacionais, de modo que as cotações da soja praticadas nos Estados Unidos são instantaneamente transmitidas para os preços recebidos pelos produtores de soja brasileiros, uma vez que a cotação interna desta commodity é fortemente influenciada por fatores externos. 
Tardelli (2013) abordou a integração entre o mercado de soja do estado do Mato Grosso e o mercado internacional. Segundo o autor, não há cointegração entre as séries de preços das principais praças produtoras de soja do Mato Grosso e a série de preços de Santos (SP), importante centro de escoamento da soja no Brasil e uma das praças de referência para o preço internacional da soja. A principal explicação para este fato, segundo o autor, é a existência de custos de transação elevados entre as praças mato-grossenses e entre estas e o porto de Santos. O autor enfatiza, dentre esses custos, os relacionados ao transporte.

No mercado doméstico de milho, Santos et al. (2007) avaliou a relação entre os preços de milho nacional e internacional no período 1996-2006 e encontrou um relacionamento no longo prazo entre essas séries de preços. Além disso, os resultados mostraram que existe uma relação de causalidade no sentido Granger unidirecional do mercado externo para o mercado interno de milho. Ademais, Caldarelli and Bacchi (2012) analisaram os principais fatores que afetaram o preço de milho no Brasil no período 1967-2008, indicando que a renda e os juros são os principais determinantes macroeconômicos do preço desse grão. Os autores usaram um modelo VEC para analisar o preço de milho no Brasil e encontraram uma forte relação entre os mercados domésticos de milho e soja. Além disso, os preços externos de milho mostraram importância no processo de formação do preço doméstico desse grão.

Mais recentemente, Tabosa, Irffi e Penna (2014) estudaram o processo de convergência dos preços de milho no mercado interno, mostrando a existência de dois clubes de convergência - o primeiro inclui os mercados de Mogiana/SP, Cascavel/PR, Chapecó/SC, Carazinho/SP, Rondonópolis/MT e São Paulo/SP e o segundo - Campo Grande/MS, Uberlândia/MG, Maringá/PR, Ponta Grossa/PR e Rio Verde/GO. Segundo os resultados, os mercados de Campinas/SP e Porto Alegre/RS não convergiram para nenhum dos clubes. Os autores concluíram que os preços do milho não estão convergindo para um determinado nível comum, logo o mercado brasileiro não apresenta convergência global.

Moratoya (2014) estudou a transmissão de preços e de volatilidade entre os mercados físicos de soja e de milho no Brasil e os preços futuros dessas commodities na CME Group, utilizando dados diários entre 2008 e 2013. Os resultados dos testes de cointegração mostraram que, para o milho, o mercado no estado de Goiás se ajusta mais rapidamente para as variações no preço futuro em Chicago, enquanto os preços em Mato Grosso se ajustem com uma velocidade mais lenta. Em relação à transmissão de volatilidade, evidências para a existência de transmissão bidirecional foi encontrada para os estados de Goiás, Mato Grosso e Paraná.

Favro, Caldarelli e Camara (2015) estimaram a elasticidade de transmissão de preços internacional-doméstico para o milho no Brasil e os resultados indicaram a existência de uma relação unidirecional entre as duas séries de preços, com o preço internacional sendo fracamente exógeno. Segundo os autores a formação do preço do milho no pais foi determinada por uma dinâmica interna, mas no mesmo tempo influenciada pelos preços internacionais.

Cruz Jr. et al. (2016) estudaram a relação entre os preços à vista e futuros de milho e soja no Brasil e nos EUA e os efeitos da expansão da produção Brasileira nessa relação nos últimos anos. Os resultados mostraram que após 2010, a integração entre os mercados spot e futuros nos dois países aumentou significativamente como resultado da produção elevada no Brasil por causa da introdução da segunda safra. Resultados semelhantes foram encontrados por Mattos e Silveira (2015), que concluíram que a introdução da safrinha no Brasil resultou numa 
mudança na sazonalidade dos preços e numa maior integração com o mercado internacional.

\section{Lei do Preço Único}

Marshall (1890) estabelece que a Lei do Preço Único (LOP, em inglês) ocorre em mercados ligados pela comercialização e arbitragem, sendo que ativos homogêneos possuem um único preço quando expostos ao mesmo câmbio e custos de transação. A LOP tem sido usada como referência na abordagem da convergência entre dois preços similares e entre índice de preços, de modo a sustentar análises de arbitragem (PIPPENGER; PHILLIPS, 2007).

Segundo Richardson (1978), as análises de arbitragem devem ser cautelosas, pois nem todas as séries cointegradas respeitam a LOP, devido a fatores influentes no preço, como os custos de transação. Mundlak e Larson (1992), ao abordarem os fundamentos da LOP para captar transmissão de preços de produtos agrícolas entre mercado mundial e doméstico, ressaltam que fatores como custos de transporte, comercialização, diferenças de qualidades entre os produtos devem ser levados em conta.

Fackler, Goodwin e Barrett (2001) argumentam que a Lei do Preço Único nem sempre é observada na prática. Segundo os autores, os preços entre duas commodities não se movem juntos se os custos de transação são distintos e voláteis. Miljkovic (1999) aponta que, além disso, análises envolvendo a Lei do Preço Único deve considerar também políticas reguladoras internas e externas, poder de mercado, heterogeneidade e perecibilidade do produto, risco de taxa de câmbio, assimetria de informação e expectativas. Com isso, na análise empírica utilizando a Lei do Preço Único, é necessário conhecer o comportamento desses drivers, alocar parâmetros que absorvam o impacto desses em relação a transmissão de preços, e abordar todas as informações necessárias para interpretar os resultados (FACKLER; GOODWIN, 2001).

Diferentes estudos empíricos buscaram testar a validade da LOP, adotando diversos métodos econométricos e de séries temporais. Chiodi (2006) analisou os preços do milho em nove estados brasileiros entre o período 1996-2004, aplicando o teste de cointegração e uma análise da equação de cointegração, a fim de verificar se a LOP é válida para esses mercados do grão. A autora mostrou que os preços de milho nos estados de São Paulo e de Minas Gerais são perfeitamente integrados com os outros mercados - um resultado que mostra a relevância desses estados na formação do preço interno do milho. Os estados de Bahia, Rio Grande do Sul e Santa Catarina se mostraram como mais independentes, por causa das importações de milho nessas regiões. Todos os estados participam na relação de cointegração, mas a LOP foi verificada somente para os preços do milho nos estados do Centro-Oeste e entre os dois estados da região Sul - Santa Catarina e Rio Grande do Sul.

Ademais, Libera (2009) analisou a transmissão entre os preços spot de soja e milho no Brasil e os preços futuros dessas commodities cotados na bolsa em Chicago, utilizando testes de causalidade de Granger e de cointegração de Johansen. $O$ autor verificou a existência de relação no longo prazo entre o preço físico e futuro de milho e entre os preços físico de soja e futuro de milho, validando os pressupostos da LOP e mostrando que os preços são integrados.

O trabalho de Sousa, Braga e Cunha (2010) seguiu a linha do trabalho do Chiodi (2006). Os autores estudaram a relação entre os preços do milho nos três estados da região Sul entre o período 2002-2009. Os autores usaram a metodologia VEC e objetivaram testar se a LOP prevaleceu nesses mercados durante o período 
analisado. Os resultados mostraram que existe cointegração entre Paraná e Rio Grande do Sul, e entre Santa Catarina e Rio Grande do Sul, porém essa relação não foi verificada para Paraná e Santa Catarina. Os autores concluíram que estes mercados não foram perfeitamente integrados e a LOP não foi completamente verificada.

Westerich Filho (2014) analisou a transmissão de preços do milho entre os estados da região Sul e Centro-Oeste e a relação entre esses preços e o preço futuro do milho cotado na BM\&FBOVESPA. O autor encontrou relação de cointegração entre todos os estados estudados e entre os preços à vista e futuro desse grão. Os resultados obtidos validaram os pressupostos da LOP e a existência de integração entre os mercados analisados.

No que tange estudos semelhantes aplicados a outras commodities, GonzálezRivera e Helfand (2001) verificaram a existência de cointegração no mercado brasileiro de arroz. Uma novidade introduzida pelos autores foi o cálculo do grau de integração entre os mercados analisados. De dezenove mercados regionais de arroz, concluiu-se que quinze são cointegrados, ou seja, podem ser considerados um único mercado, segundo a definição dos autores. Dentre estes, foram ainda identificados alguns mercados que tem uma influência maior sobres os outros e que, na formulação de políticas públicas, deveriam receber mais atenção.

No mercado de soja, Souza e Campos (2008) verificaram a validade da Lei do Único Preço entre os estados do Paraná, Rio Grande do Sul e Mato Grosso. Concluiuse que estes mercados são cointegrados e que a Lei do Preço Único se aplica. Além disso, entre esses estados ocorre a transmissão de preços no longo prazo.

Da Silva, Fracaroli e Sobel (2013) analisaram a transmissão de preços entre as praças produtoras de soja no estado do Mato Grosso. Os autores utilizaram o modelo VEC threshold e o modelo SETAR, que são modelos que captam custos de transação. Entre os resultados, verificou-se que entre as praças analisadas não ocorre transmissão de preços, embora sejam geograficamente próximas. Assim sendo, estes refletem claramente os custos de transação

\section{METODOLOGIA}

\section{Dados}

O presente modelo testará a hipótese da existência de uma relação causal entre os preços da unidade, saca de milho de $60 \mathrm{~kg}$, praticados nas praças produtoras de Canarana, Diamantino, Lucas do Rio Verde, Rondonópolis, Sapezal, Sorriso e Paranaguá. As séries de preços envolvem os preços disponíveis no intervalo de tempo de janeiro de 2009 a abril de 2015, elencando seis anos para análise. As praças selecionadas do Mato Grosso (advêm) do nível de importância dessas, elencando alta produção do grão, localização e grau de comercialização. Os dados das praças produtoras do Mato Grosso foram disponibilizados pelo Instituto Mato Grossense de Economia Agropecuária (IMEA) e os dados de Paranaguá pelo Centro de Estudos Avançados em Economia Aplicada (CEPEA).

\section{Teste de Raiz Unitária}

Após a obtenção das séries temporais, realizou-se a análise de testes de estacionariedade para verificar a existência ou não de raízes unitárias nas séries 
empregadas no modelo, através dos testes de Dickey-Fullher aumentado (ADF), Phillips-Peron (PP) e Kwiatkowski, Phillips, Schmidt e Shin (KPSS).

Conforme apresentado por Enders (2003), os choques em uma série de tempo estacionária são necessariamente temporários, pois ao longo do tempo ocorre dissipação dos efeitos dos choques e a série reverte-se para seu nível médio de longo prazo. Com isso, previsões de longo prazo de uma série estacionária convergem para média incondicional da mesma.

Uma série temporal é estacionária se sua média, variância e autocovariância permanecem as mesmas independentemente do período de tempo em que sejam medidas. Nesse caso, a série temporal é considerada um processo integrado de ordem zero. Caso contrário, a série temporal é chamada de não estacionária, sendo conhecida como processo integrado de ordem I(d), em que d corresponde ao número de vezes que a série original deve ser diferenciada para que se transforme em um processo I(0). As séries intituladas estacionárias seguem as propriedades de Enders (2003).

O teste Dickey-Fuller aumentado (ADF) considera a possibilidade de que o termo de erro seja autocorrelacionado, pressupondo que a regressão utilizada para a realização do teste deve ser modificada de forma a incluir termos defasados em primeira diferença e possibilitar a obtenção do erro.

\section{Teste de Causalidade de Granger}

O Teste de Causalidade de Granger (Granger e C.W.J., 1969) é um teste de hipótese realizado para determinar se uma série temporal pode ser útil no processo de previsão de uma outra série. Esse teste corresponde a algumas técnicas clássicas para a aferição de relações de preços entre mercados futuros e físicos, e que foram aplicadas em inúmeras pesquisas ao redor do mundo.

Considere duas séries de tempo X e Y. O teste de causalidade de Granger assume que a informação relevante para a predição das respectivas variáveis $X$ e $Y$ está contida apenas nas séries de tempo sobre essas duas variáveis. Dessa forma, uma série de tempo estacionária $X$ causa, no sentido de Granger, uma outra série estacionária $Y$ se melhores predições estatisticamente significantes de $Y$ podem ser obtidas ao incluirmos valores defasados de $X$ aos valores defasados de $Y$.

O teste de causalidade de Granger para os preços de milho em Paranaguá e nas praças de Mato Grosso baseia-se nas seguintes equações:

$$
\begin{aligned}
& P_{P A R, t}=\sum_{j=1}^{m} a_{j} P_{P A R, t-j}+\sum_{j=1}^{m} b_{j} P_{i, t-j}+\varepsilon_{1 t} \\
& P_{i, t}=\sum_{j=1}^{m} c_{j} P_{i, t-j}+\sum_{j=1}^{m} d_{j} P_{P A R, t-j}+\varepsilon_{2 t}
\end{aligned}
$$

em que $P_{P A R, t}$ é a série de preços de Paranaguá no tempo "t", $P_{i, t}$ é a série de preços da praça $i$ no tempo $t$ no Mato Grosso; e $P_{P A R, t-j}$ e $P_{i, t-j}$ são os preços defasados $j$ períodos.

\section{Modelo Vector Error Correction Model (VECM)}

O estudo da transmissão de preços entre commodities homogêneas tem atraído interesses de muitos economistas da parte agrícola, segundo Meyer (2004), de modo que as técnicas de análise de transmissão de preços têm se desenvolvido cada vez mais estatisticamente. 
O primeiro estudo de transmissão de preços de mercados agrícolas utilizando métodos de cointegração foi realizado por Ardeni (1989). Hoje em dia, boa parte de estudos empíricos de transmissão de preços utilizam métodos de cointegração e, também, o vector error correction model (VECM), que compõe um modelo reparametrizado do vetor autorregressivo (VAR), o VECM é capaz de captar interações entre preços internacionais e domésticos.

A aplicação do VECM depende da composição e relação entre as séries temporais de preços analisadas, caso as series sejam cointegradas, ou seja, possuem uma relação de longo prazo entre elas, o VECM é a ferramenta econométrica eficaz para captar transmissão de preços em nível. Caso as séries não sejam cointegradas, a relação pode ocorrer no curto prazo, nesse caso o VAR se mostra eficiente para a análise de transmissão de preços (Engle e Granger, 1987).

O VECM é a ferramenta econométrica mais utilizada para trabalhos empíricos de transmissão de preços, porém possuí algumas características restritivas. Hassouneh (2012b) elencou duas características de linearidade na equação utilizada no VECM, a primeira é linear no sentido de que todos os parâmetros do modelo são constantes em toda amostra do período analisado. Segunda, as variáveis dependentes reagem linearmente às mudanças das variáveis independentes. A primeira característica é prejudicial a analises de transmissão de preços entre mercado nacional e internacional, de modo que esses parâmetros não podem ser constantes em todo o tempo analisado. Uma mudança na política de comércio internacional pode alterar a natureza da relação de transmissão de preços. Desse modo, o VECM pode ser ajustado a "regimes" dentro do intervalo de tempo analisado, de modo a discriminar dois contextos econômicos diferentes de série de preços, para que o modelo VECM seja aplicado com eficiência.

O modelo VECM utilizado nesse trabalho se baseia na seguinte equação:

$$
\Delta P_{t}=\zeta_{1} \Delta P_{t-1}+\zeta_{2} \Delta P_{t-2}+\cdots+\zeta_{p-1} \Delta y_{t-p+1}-B z_{t-1}+\alpha+\varepsilon_{t}
$$

em que $p$ é o número de defasagens, $\Delta P_{t}$ é o vetor de preços em primeira diferença nas praças de milho, $\zeta_{i}$ são os coeficientes de ajustamento no curto prazo, $B$ é a matriz de coeficientes de ajustamento no longo prazo, $z_{t-1}$ é o termo de correção de erro. Considerando as seis praças de Mato Grosso, o modelo para a primeira praça específica fica:

$$
\begin{gathered}
\Delta p_{1, t}=\sum_{i=1}^{p-1} \zeta_{11}(i) \Delta p_{1, t-i}+\sum_{i=1}^{p-1} \zeta_{12}(i) \Delta p_{2, t-i}+\cdots+\sum_{i=1}^{p-1} \zeta_{16}(i) \Delta p_{6, t-i}- \\
\sum_{j=1}^{h} b_{1, j} z_{j, t-1}+\alpha_{1}+\varepsilon_{1 t}
\end{gathered}
$$

\section{RESULTADOS E DISCUSSÃO}

As séries diárias referentes ao preço de milho negociado nas praças de Paranaguá, Diamantino, Rondonópolis, Sorriso, Canarana, Lucas do Rio Verde e Sapezal estão dispostas na figura 1. As séries estão em nível e correspondem ao período que vai de 05 de janeiro de 2009 a 07 de abril de 2015, totalizando 1500 observações. É importante destacar que os resultados encontrados neste trabalho são válidos para a primeira safra e segunda safra (safrinha) de milho, a cada ano. 
Figura 1 - Comportamento dos preços do milho nas praças de comercialização do Mato Grosso selecionadas (2009-2015), preços em nível, em R\$/saca.

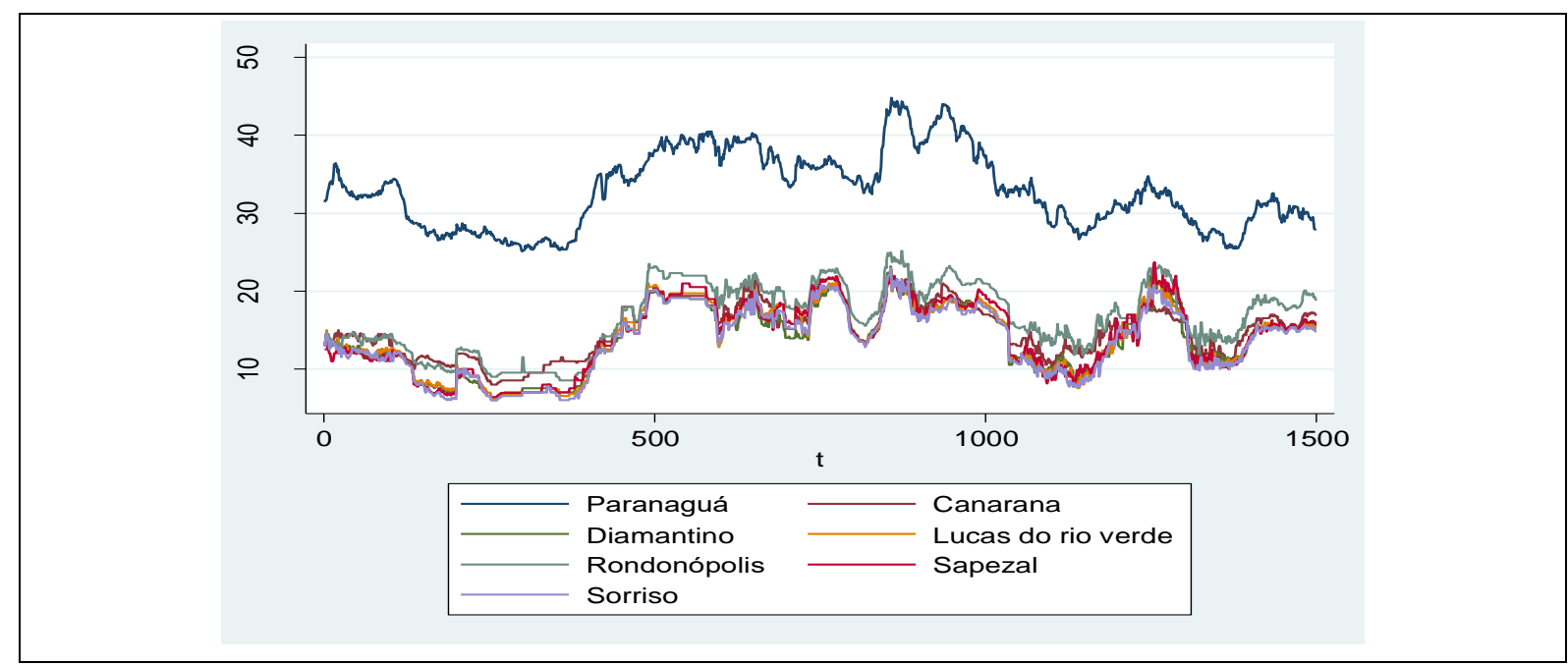

Fonte: (IMEA, 2015), elaborado pelos autores.

As estatísticas descritivas relativas às séries de preço utilizadas neste trabalho estão dispostas na Tabela 1. Como a tabela mostra, o preço médio mais alto igual a 32,91 reais por saca foi registrado em Paranaguá. Para as praças de Mato Grosso o preço médio no período observado ficou entre 13 e 17 reais por saca. Quanto ao desvio-padrão, a tabela mostra que os preços de todas as praças têm mais ou menos a mesma variabilidade. A curtose, que mostra se a distribuição dos dados coincide com a distribuição normal, é positiva para todas as praças, que significa que a distribuição de todos os preços é mais repicada do que a distribuição normal. Quanto à assimetria, os dados mostram que todas as praças de Mato Grosso têm uma distribuição de preços assimétrica, com uma longa cauda para a esquerda, enquanto o preço de Paranaguá mostrou uma distribuição assimétrica com uma longa cauda para a direita.

Tabela 1 - Estatísticas Descritivas das séries de preço de milho, de janeiro de 2009 a abril de 2015.

\begin{tabular}{lrrrrrrr}
\hline & Paranaguá & Canarana & Diamantino & $\begin{array}{l}\text { Lucas do } \\
\text { Rio Verde }\end{array}$ & Rondonópolis & Sapezal & Sorriso \\
\hline Média & 32,91 & 15,03 & 13,88 & 14,00 & 16,58 & 14,14 & 13,59 \\
Máximo & 44,81 & 23 & 23,2 & 22,8 & 25,2 & 23,73 & 23 \\
Mínimo & 25,09 & 8 & 6 & 6,2 & 8,5 & 6,4 & 6 \\
Desvio-padrão & 4,87312 & 3,4151 & 4,22 & 4,3472 & 4,3625 & 4,5118 & 4,3488 \\
Assimetria & 0,3247 & $-0,0442$ & $-0,0995$ & $-0,1462$ & $-0,1572$ &,- 0728 &,- 1563 \\
Curtose & 2,1631 & 2,0310 & 1,9122 & 1,8190 & 1,8962 & 1,8139 & 1,867 \\
Observações & 1500 & 1500 & 1500 & 1500 & 1500 & 1500 & 1500 \\
\hline
\end{tabular}

Fonte: Dados da pesquisa.

\section{Estacionariedade e Causalidade}

Para testar a estacionariedade das séries de preço utilizou-se um teste de Dickey-Fuller Aumentado (ADF). Na Tabela 2 estão os resultados do teste para as 
séries diárias em nível e na Tabela 3 os resultados para as séries em primeira diferença. Constatou-se que as séries de preço de milho de todas as praças de negociação contêm raiz unitária, ou seja, são integradas de ordem 1. Após a primeira diferenciação essas mesmas séries se tornam estacionárias, ou seja, integradas de ordem zero.

Tabela 2 - Teste de raiz unitária para os preços em nível.

\begin{tabular}{lccccc}
\hline & $\mathbf{Z}$ & $\mathbf{1 \%}$ & $\mathbf{5 \%}$ & $\mathbf{1 0 \%}$ & $\begin{array}{c}\text { Ordem de } \\
\text { Integração }\end{array}$ \\
\hline Paranaguá & -1.799 & -3.43 & -2.86 & -2.57 & $\mathrm{I}(1)$ \\
Canarana & -2.197 & -3.43 & -2.86 & -2.57 & $\mathrm{I}(1)$ \\
Diamantino & -1.881 & -3.43 & -2.86 & -2.57 & $\mathrm{I}(1)$ \\
Lucas do Rio Verde & -1.986 & -3.43 & -2.86 & -2.57 & $\mathrm{I}(1)$ \\
Rondonópolis & -1.845 & -3.43 & -2.86 & -2.57 & $\mathrm{I}(1)$ \\
Sapezal & -1.972 & -3.43 & -2.86 & -2.57 & $\mathrm{I}(1)$ \\
Sorriso & -1.943 & -3.43 & -2.86 & -2.57 & $\mathrm{I}(1)$ \\
\hline
\end{tabular}

Fonte: Dados da pesquisa

Tabela 3 - Teste de raiz unitária para os preços primeira diferença.

\begin{tabular}{lccccc}
\hline & $\mathbf{Z}$ & $\mathbf{1 \%}$ & $\mathbf{5 \%}$ & $\mathbf{1 0 \%}$ & Ordem de Integração \\
\hline Paranaguá & -15.589 & -3.43 & -2.86 & -2.57 & $\mathrm{I}(0)$ \\
Canarana & -17.665 & -3.43 & -2.86 & -2.57 & $\mathrm{I}(0)$ \\
Diamantino & -16.83 & -3.43 & -2.86 & -2.57 & $\mathrm{I}(0)$ \\
Lucas do Rio Verde & -16.944 & -3.43 & -2.86 & -2.57 & $\mathrm{I}(0)$ \\
Rondonópolis & -16.981 & -3.43 & -2.86 & -2.57 & $\mathrm{I}(0)$ \\
Sapezal & -16.774 & -3.43 & -2.86 & -2.57 & $\mathrm{I}(0)$ \\
Sorriso & -16.401 & -3.43 & -2.86 & -2.57 & $\mathrm{I}(0)$ \\
\hline
\end{tabular}

Fonte: Dados da pesquisa

O teste de causalidade de Granger aponta uma relação de causalidade unidirecional, partindo da praça de Paranaguá em relação às praças matogrossenses. Os resultados do teste estão dispostos nas Tabelas 4 e 5 . A Tabela 4 mostra que os preços praticados em Paranaguá aumentam a capacidade de previsão dos preços nas praças de Canarana, Diamantino, Lucas do Rio Verde, Rondonópolis, Sapezal e Sorriso. A Tabela 5 confirma a relação unidirecional, ou seja, os preços praticados nas praças mato-grossenses não aumentam a capacidade de previsão dos preços praticados em Paranaguá. Uma única exceção se faz em relação à praça de Canarana, sendo possível rejeitar ao nível de 5\% a hipótese nula de que os preços praticados em Canarana não ajudam na previsão dos preços de Paranaguá, o que não era esperado. 
Tabela 4 - Teste de Causalidade de Granger

\begin{tabular}{lccc}
\hline \multicolumn{1}{c}{ Hipótese nula } & chi' $^{\mathbf{2}}$ & $\mathbf{P < | c h \mathbf { 2 } ^ { 2 } |}$ & \\
\hline Paranaguá não Granger-causa Canarana & 27,066 & 0,000 & Rejeita \\
Paranaguá não Granger-causa Diamantino & 21,757 & 0,000 & Rejeita \\
Paranaguá não Granger-causa Lucas do Rio Verde & 22,561 & 0,000 & Rejeita \\
Paranaguá não Granger-causa Rondonópolis & 24,119 & 0,000 & Rejeita \\
Paranaguá não Granger-causa Sapezal & 27,186 & 0,000 & Rejeita \\
Paranaguá não Granger-causa Sorriso & 21,628 & 0,000 & Rejeita \\
\hline
\end{tabular}

Fonte: Dados da pesquisa

Tabela 5 - Teste de Causalidade de Granger

\begin{tabular}{lccc}
\hline \multicolumn{1}{c}{ Hipótese nula } & chi $^{2}$ & $\mathbf{P}<\mid \mathbf{c h i} \mathbf{2}^{2}$ & \\
\hline Canarana não Granger-causa Paranaguá & 5,0903 & 0,024 & Rejeita \\
Diamantino não Granger-causa Paranaguá & 3,304 & 0,069 & Não Rejeita \\
Lucas do Rio Verde não Granger-causa Paranaguá & 3,3513 & 0,067 & Não Rejeita \\
Rondonópolis não Granger-causa Paranaguá & 0,43594 & 0,509 & Não Rejeita \\
Sapezal não Granger-causa Paranaguá & 1,5551 & 0,212 & Não Rejeita \\
Sorriso não Granger-causa Paranaguá & 3,4076 & 0,065 & Não Rejeita \\
\hline
\end{tabular}

Fonte: Dados da pesquisa

\section{Cointegração}

Após a aplicação dos testes de raiz unitária e do teste de causalidade de Granger, o próximo passo foi a aplicação do teste de cointegração de Johansen. $O$ objetivo ao aplicar este teste foi verificar a existência de relações de longo prazo entre as séries de preço de Paranaguá e das praças do Mato Grosso. O número de defasagens foi definido de acordo com os critérios de Akaike e Schwarz. A Tabela 6 mostra os resultados do teste de cointegração feito entre Paranaguá e as praças matogrossenses. Apenas as séries de preços relativas às praças de Canarana, Lucas do Rio Verde e Sapezal estão cointegradas com a série de preços de Paranaguá, mantendo portando, uma relação de longo prazo entre essas praças e Paranaguá.

Este resultado se assemelha ao resultado encontrado por Tardelli (2013). O autor encontrou pouca relação entre os preços de soja no estado do Mato Grosso e o preço do milho no porto de exportação. Mais especificamente, o autor não encontrou nenhuma relação de cointegração entre diversas praças, entre elas Rondonópolis, Diamantino, Lucas do Rio Verde, Sorriso e Canarana, e Santos (SP), outro grande porto de escoamento do cereal. A principal razão apontada por Tardelli (2013) foi a existência de elevados custos de transação entres as praças analisadas. Os resultados encontrados por Tardelli (2013) para a soja podem ser estendidos para o milho, pois segundo Chiodi (2006), a produção de milho no estado do Mato Grosso se desenvolveu em função da produção de soja. As duas culturas compartilham estruturas de produção e transporte até os portos de escoamento. 
Tabela 6 - Teste do cointegração de Johansen para as praças do Mato Grosso e Paranaguá

\begin{tabular}{lccc}
\hline Pares & r0=0 & r1=1 & Valor crítico 5\% \\
\hline Canarana-Paranaguá & & $1,83^{*}$ & 3,76 \\
Diamantino-Paranaguá & & $1,68^{*}$ & 3,76 \\
Lucas do Rio Verde - Paranaguá & $14,87^{*}$ & & 15,41 \\
Rondonópolis-Paranaguá & $15,54^{*}$ & & 15,41 \\
Sapezal-Paranaguá & $14,93^{*}$ & & 15,41 \\
Sorriso-Paranaguá & $14,33^{*}$ & & 15,41 \\
\hline
\end{tabular}

Fonte: Dados da pesquisa

O resultado do teste de cointegração entre as praças de Mato Grosso está disposto na Tabela 7. Existe cointegração entre todas as praças analisadas, havendo, portando, relações de longo prazo entre todas elas. Assim sendo, é possível afirmar que o milho comercializado nas seis praças analisadas é substituto entre si e que existe certo nível de arbitragem na comercialização, o que faz com os preços praticados sejam próximos.

Tabela 7 - Teste do cointegração de Johansen para as praças do Mato Grosso Canarana, Diamantino, Lucas do Rio Verde, Rondonópolis, Sapezal e Sorriso.

\begin{tabular}{ccc}
\hline Número de vetores cointegrantes & Traço & Valor Crítico a 5\% \\
\hline $\mathbf{0}$ & 290,97 & 94,15 \\
$\mathbf{2}$ & 190,22 & 68,52 \\
$\mathbf{3}$ & 119,59 & 47,21 \\
$\mathbf{4}$ & 63,99 & 29,68 \\
$\mathbf{5}$ & 28,67 & 15,41 \\
$\mathbf{6}$ & $2,50^{*}$ & 3,76
\end{tabular}

Fonte: Dados da pesquisa

\section{Elasticidades de transmissão de preços entre Paranaguá e as praças de Mato Grosso}

Conforme os resultados da Tabela 7, apenas as séries de preço das praças de Canarana e Diamantino mantêm uma relação de longo prazo com Paranaguá. As séries das demais praças mato-grossenses não são cointegradas com Paranaguá, não mantendo com ela, portanto, nenhuma relação de longo prazo. Diante deste resultado, a análise da transmissão de preços foi feita utilizando um modelo VECM para os pares Canarana-Paranaguá e Diamantino-Paranaguá. Utilizou-se as séries de preço em logaritmos neperianos, de forma que os resultados dos modelos VECM e VAR sejam exatamente as elasticidades de transmissão de preços. Neste caso definiu-se, pelo critério SBIC, que o número de lags a ser usado é 1 .

As elasticidades de transmissão de preços de longo prazo entre as praças de Paranaguá e as praças mato-grossenses estão na Tabela 8 e as relações de longo prazo entre as próprias praças mato-grossenses estão na tabela 9. As variáveis dependentes são as praças de Mato Grosso e a variável explicativa é Paranaguá. 
Tabela 8 - Elasticidades de transmissão de preços de Paranaguá para as praças do Mato Grosso

\begin{tabular}{ccccccc}
\hline & Canarana & Diamantino & $\begin{array}{c}\text { Lucas do Rio } \\
\text { Verde }\end{array}$ & Rondonópolis & Sapezal & Sorriso \\
\hline Paranaguá & $-1,466^{* * *}$ & $-2,108^{* * *}$ & N.I. & N.I. & N.I. & N.I.
\end{tabular}

${ }^{* *}$ significativo a $5 \%,{ }^{* * *}$ significativo a $1 \%$, N.I.: não cointegradas.

Fonte: dados da pesquisa

As elasticidades de transmissão de preço, na direção de Paranaguá para as praças de Canarana e Diamantino podem ser consideradas altas. A maior elasticidade de transmissão foi registrada de no par Diamantino-Paranaguá. Uma variação de 10\% no preço do milho em Paranaguá causa uma variação de $21,08 \%$ no preço do milho em Diamantino e uma variação de $14,66 \%$ no preço do milho em Canarana. A baixa integração entre o preço do milho nos mercados de Mato Grosso e o mercado de Paranaguá (portanto, com o mercado internacional) era esperada.

Numa análise comparativa com outros trabalhos, Tardelli (2013) encontrou um resultado muito semelhante a este ao analisar os mercados de Mato Grosso e o mercado de Santos (outra proxy para o mercado internacional). O autor atribui a baixa integração à precária integração física entre as praças produtores e o porto exportador, o que se verifica na péssima qualidade das rodovias, na quase inexistência de transporte ferroviário e hidroviário suficientes. Portanto, os altos custos de transporte entre as praças mato-grossenses e os portos de exportação do milho são um grande obstáculo na integração de preços. Também neste contexto, Moratoya (2014) verifica a existência de uma transmissão unidirecional de choques de preço no que partem do mercado internacional (o autor utilizou como proxy os preços futuros da Chicago Board of Trade) para o mercado de milho do estado do Mato Grosso. Em sentido mais amplo, Santos et a. (2007) confirmam a importância do mercado internacional de milho na formação dos preços do mercado interno de milho brasileiro, ou seja, os autores verificam a existência de transmissão de preços do mercado internacional para o mercado nacional de milho.

\section{Elasticidades de transmissão de preços entre as praças de Mato Grosso}

Afim de analisar a transmissão de preços entre as praças do Mato Grosso, optou-se por um modelo de correção de erros (VECM). A opção por este modelo é justificada pelo resultado do teste da Tabela 7 , que mostra a existência de cointegração entre as séries de preços de todas as praças do estado. $O$ número de lags igual a 4 foi definido com base nos critérios de LR, FPE e AIC. As elasticidades de transmissão de preços de longo prazo entre as praças mato-grossenses estão na Tabela 9.

Os preços do milho nas praças mato-grossenses são correlacionados, sendo possível estabelecer relações de sentido e causalidade. Isso faz com que esses preços sejam fontes de informações confiáveis e podem ser levados em consideração para a tomada de decisão dos agentes. 
Tabela 9 - Elasticidades de transmissão de preços entres as praças do Mato Grosso

\begin{tabular}{lcccccc}
\hline & Canarana & Diamantino & $\begin{array}{c}\text { Lucas do } \\
\text { Rio Verde }\end{array}$ & Rondonópolis & Sapezal & Sorriso \\
\hline Canarana & & $-1,42202$ & $-1,462848$ & $-1,227816$ & $-1,4909$ & $-1,527$ \\
Diamantino & $-0,70323$ & & $-1,028075$ & $-0,854742$ & $-1,0461$ & $-1,070$ \\
Lucas do Rio & $-0,6836$ & $-0,972692$ & & $-0,8313963$ & $-1,0175$ & $-1,041$ \\
Verde & & & & & & \\
Rondonópolis & $-0,81445$ & $-1,169943$ & $-1,202796$ & & $-1,2240$ & $-1,251$ \\
Sapezal & $-0,67073$ & $-0,955863$ & $-0,9827488$ & $-0,8169786$ & & $-1,023$ \\
Sorriso & $-0,65451$ & $-0,934359$ & $-0,960603$ & $-0,7988091$ & $-0,977$ & \\
\hline
\end{tabular}

Fonte: dados da pesquisa

A partir da Tabela 9 constata-se que o mercado de milho no Mato Grosso se encaixa naquilo que González-Rivera e Helfand (2001) classificam como um mercado integrado, isto é, um mercado que todas as localidades transacionam a mesma commodity, neste caso o milho, e que mantenham relações informacionais de longo prazo, o que foi verificado pelo VECM.

Trata-se, portanto, de um sistema em que cada região reage a qualquer desiquilíbrio de outra região. Os autores classificam essa situação de interdependência extrema. Empiricamente, essa situação se verifica caso os termos de erros de todas as equações do modelo VECM sejam significativos. No caso deste trabalho, todos os termos de erro são significativos a, no máximo, $5 \%$, o que pode ser verificado na tabela 10.

Relações semelhantes de integração entre mercados regionais de milho vem sendo identificadas no Brasil (Westerich Filho 2014; Tabosa et al. 2014; Sousa et al. 2010; Chiodi 2006). Em linhas gerais, os autores confirmam a existência, em maior ou menor grau, de cointegração no mercado de milho entre os estados das regiões Sul, Sudeste e Centro-Oeste, muito embora a integração dos mercados não se verifique necessariamente entre todos os estados. Até este ponto, não foram encontrados trabalhos que analisassem os mercados de milho do estado do Mato Grosso.

As elasticidades de transmissão do preço de milho especialmente altas (elásticas) nos pares Canarana-Diamantino, Canarana-Lucas do Rio Verde, Canarana-Sapezal, Canarana-Sorriso e Canarana -Rondonópolis. Este é um resultado que difere de outros estudos que feitos até este ponto e pode indicar uma mudança na dinâmica do mercado mato-grossense de milho. De acordo com o IMEA (2016), a região Nordeste do estado do Mato Grosso, na qual se situam as cidades de Canarana e Querência, é a que vem liderando o crescimento do cultivo de milho no estado. Nas últimas cinco safras, a região dobrou a área plantada de milho e até a safra 2021/2022 espera-se que a área plantada dobre mais uma vez. Apesar do crescimento, a área de cultivo de milho na região Norte ainda é a menor entre todas as regiões do Mato Grosso.

Observou-se também elasticidades de transmissão relativamente altas nos pares Rondonópolis-Diamantino, Rondonópolis-Lucas do Rio Verde, RondonópolisSapezal e Rondonópolis-Sorriso. A transmissão de preços de Rondonópolis para as demais praças mato-grossenses é justificável pela importância desta cidade na logística do escoamento do milho, principalmente para o porto de Santos (SP). Em análise para a soja no Mato Grosso, Tardelli (2013) encontra resultado semelhante.

Praças produtoras com relativa proximidade geográfica, como é o caso de Diamantino, Lucas do Rio Verde e Sapezal, apresentam elasticidades de transmissão 
de preço próximas à unidade. Tal resultado pode ser explicado pela proximidade territorial das praças, que implica menores custos de transação entre elas. Nesse contexto, a Tabela 10 mostra os termos de erro do VECM, que informam as velocidades de ajustamento à tendência de longo prazo a partir de choques de curto prazo.

Tabela 10 - Termos de erros estimados do VECM

\begin{tabular}{|c|c|c|c|c|c|c|}
\hline & Canarana & Diamantino & $\begin{array}{l}\text { Lucas do } \\
\text { Rio Verde }\end{array}$ & $\begin{array}{l}\text { Rondonópoli } \\
\text { s }\end{array}$ & Sapezal & Sorriso \\
\hline Canarana & & $-0,0247^{* * *}$ & $-0,0233^{* * *}$ & $-0,0237^{* * *}$ & $-0,0283^{* * *}$ & $-0,015^{\star *}$ \\
\hline Diamantino & $-0,0360^{* * *}$ & & $-0,0755^{* * *}$ & $-0,0405^{\star * *}$ & $-0,0631^{* * *}$ & $-0,063^{* * *}$ \\
\hline $\begin{array}{l}\text { Lucas do Rio } \\
\text { Verde }\end{array}$ & $-0,0335^{\star * *}$ & $-0,0576^{* * *}$ & & $-0,0373^{* * *}$ & $-0,058^{* * *}$ & $-0,0855^{\star * *}$ \\
\hline Rondonópolis & $-0,0189^{* *}$ & $-0,0277^{* * *}$ & $-0,0246^{* *}$ & & $-0,031^{* * *}$ & $-0,023^{* *}$ \\
\hline Sapezal & $-0,0275^{\star * *}$ & $-0,0431^{* * *}$ & $-0,0493^{* * *}$ & $-0,0305^{\star \star *}$ & & $-0,054^{* * *}$ \\
\hline Sorriso & $-0,0300^{* * *}$ & $-0,0611^{* * *}$ & $-0,0880 * * *$ & $-0,0338^{* * *}$ & $-0,057^{* * *}$ & \\
\hline
\end{tabular}

${ }^{* *}$ significativo a $5 \%$, demais significativos a $1 \%$

Fonte: dados da pesquisa

A partir da Tabela 10 constata-se que variações de curto prazo na trajetória do preço do milho entre Lucas do Rio Verde e Sorriso são corrigidas de um dia para o outro em $8,8 \%$. Termos de erro relativamente altos são encontrados particularmente entre as praças de Sorriso, Diamantino e Lucas do Rio Verde. Em geral, os desequilíbrios de curto prazo nas trajetórias do preço do milho dessas três praças são corrigidos de 6,1 a $8,8 \%$ de um dia para o outro. É interessante notar que, dentre todas as praças, Sorriso, Lucas do Rio Verde e Diamantino são as que estão geograficamente mais próximas umas das outras.

\section{CONCLUSÃO}

O objetivo principal desse trabalho foi analisar a transmissão de preços entre Paranaguá, que reflete o comportamento do mercado externo, e as principais praças produtoras de milho no estado de Mato Grosso, usando dados diários para o período entre 2009 e 2015. Mais especificamente, a ferramenta econométrica VECM foi utilizada para identificar as relações existentes no curto e no longo prazo entre os preços de milho no Estado, que é um dos maiores produtores de grãos do Brasil.

Os resultados mostram que o preço em Paranaguá Granger-causa os preços nas todas as praças em Mato Grosso, enquanto essa causalidade no sentido oposto não foi encontrada. Porém, o teste de cointegração mostrou que existe uma relação no longo prazo apenas entre Paranaguá e duas das praças mato-grossenses Canarana e Diamantino. Essa baixa integração entre o mercado interno de milho e o porto exportador pode ser atribuída à precária integração física entre esses lugares, que implica altos custos de transporte.

A relação de cointegração foi encontrada entre todas as praças de milho analisadas, assim mostrando que o setor de milho no Mato Grosso pode ser classificado como um mercado integrado com alta interdependência, onde o preço de cada praça reage a qualquer desequilíbrio dos preços nas outras praças. 
O conhecimento das relações entre os preços de uma commodity em locais diferentes tem importância para todos os agentes na cadeia produtiva dessa commodity. Então, os resultados de uma análise, como a do presente trabalho, a transmissão entre os preços de milho em praças diferentes, seria aplicável para os produtores na região, reagindo às variações nos preços do seu produto. Além disso, a identificação das praças produtoras mais importantes e que tem mais influências sobre as outras é de fundamental importância para a eficácia de políticas públicas para o setor.

Como sugestão para trabalhos futuros, recomenda-se um aprofundamento no estudo da transmissão de preço do milho no estado do Mato Grosso utilizando modelos que captem os custos de transação, como VEC threshold e SETAR, visto que esses custos são uma variável de fundamental importância nos preços de produtos agrícolas no Centro-Oeste em geral, e no Mato Grosso, em particular.

\section{REFERÊNCIAS}

ALLEN, E.; VALDES, C. Brazil's corn industry and the effect on the seasonal pattern of U.S. corn exports. AES-93 Economic research Service/USDA, 2016.

ARDENI, P.G. Does the Law of One Price Really Hold for Commodity Prices? American Journal Of Agricultural Economics . Vol. 71, n.3, p. 661 - 669, 1989.

CALDARELLI, C. E.; BACCHI, M. R. P. Fatores de influência no preço do milho no Brasil. Nova Economia, Belo Horizonte, v. 22, n. 1, p. 141-164, 2012.

CEPEA, Centro de Estudos Avançados em Economia Aplicada. Acesso em: Janeiro de 2016. Disponível em: http://www.cepea.esalq.usp.br/.

CHIODI, L. Integração espacial no mercado brasileiro de milho. 2006. 90 f. Dissertação (Mestrado em Economia Aplicada) - Universidade de São Paulo, Escola Superior de Agricultura "Luiz de Queiroz", Piracicaba.

CONAB, Companhia Nacional de Abastecimento. Acompanhamento da safra de grãos. Publicado em: Agosto de 2015. Acesso em: http://www.conab.gov.br/conteudos.php?a=1253\&.

CORONEL, D.; AMORIM, A.; SOUSA, E.; LIMA, J. Integração e Transmissão de Preços entre os Mercado de Trigo Argentino e Internacional: Pesquisa \& Debate. Vol. 21, n. 2, p. 279-305, 2010.

CRUZ JR, J. C.; CAPITANI, D. H. D.; SILVEIRA, R. L. F.; URSO, F. S. P.; MARTINES FILHO J. G. The effect of Brazilian corn and soybean crop expansion on price and volatility transmission. Selected paper prepared for presentation, In: AGRICULTURAL \& APPLIED ECONOMICS ASSOCIATION ANNUAL MEETING, Boston, MA, Jul. 31 Aug. 02, 2016.

DA SILVA, M.M.; FRASCAROLI, B.F.; SOBEL, T.F. Transmissão de preços e custos de transação no mercado de soja mato-grossense: uma abordagem por modelos threshold. Revista de Economia e Agronegócio. Vol. 11, v.2 , pp. 185-210, 2013. 
ENDERS, W. Applied econometric time series. New York: John Wiley \& Sons, 1995. $433 p$.

ENGLE, R.F.; GRANGER, C.W.J. Cointegration and Error,correction: Representation, Estimation and Testing. Econometrica. Vol. 55, n.2, p. 251-276, 1987.

FACKLER, P.L.; GOODWIN, B.K. Spatial Price Analysis. In: B. L. Gardner, and G. C.Rausser (eds). Handbook of Agricultural Economics, Elsevier: 972-1025, 2001.

FAVRO, J.; CALDARELLI, C. E.; CAMARA, M. R. G. Modelo de análise da oferta de exportações de milho brasileira: 2001 a 2012. RESR, Piracicaba - SP, v. 53, n. 3, p. 455-476, jul./set. 2015.

FULLER, W.A. Introduction to statistical time series. 2.ed. New York: John Wiley \& Sons, 1976.

GOODWIN, B.K. and PIGGOT N.E. Spatial Market Integration in the Presence of Threshold Effects. American Journal of Agricultural Economics. Vol. 83, n.2., p. 302-317, 2001

GONZÁLEZ-RIVERA, G; HELFAND, S.M. The extent, pattern, and degree of market integration: a multivariate approach for the Brazilian rice market. American Journal of Agricultural Economics. Vol. 83, n. 3, p. 576-592, 2001.

GREB, F., KRIVOBOKOVA, T.; CRAMON-TAUBADEL, S.; MUNK, A. (2011). On Threshold Estimation in Threshold Vector Error Correction Models. In: European Association of Agricultural Economists 2011 Congress, Zurich, 2011.

HASSOUNEH, I.; SERRA, T; GOODWIN, B.K.; GIL, J.M. Non-Parametric and Parametric Modeling of Biodiesel - Sunflower Oil - Crude Oil Price Relationships. Energy Economics. Vol. 34, n.5, p. 1507-1513, 2012a

HASSOUNEH, I.; CRAMON-TAUBADEL, S.; SERRA, T.; GIL, J.M. Recent Developments in the Econometric Analysis of Price Transmission. Working Paper, Transparency of Food Pricing, 2012b

IMEA, Instituto Mato-Grossense de Economia Agropecuária. Séries de dados. Acesso em: Agosto de 2015. Disponível em: http://www.imea.com.br/site/precos.php

KAPPES, C. Sistemas de cultivo de milho safrinha no Mato Grosso. In: XII SEMINÁRIO NACIONAL Estabilidade e Produtividade, Embrapa, Dourados, MS, 2628 de novembro, 2013.

LIBERA, A. A. D. Integração entre os mercados de milho e soja: uma análise através de transmissão de preços. 2009. 156 f. Dissertação (Mestrado em Agronegócios) - Universidade federal do Rio Grande do Sul, Porto Alegre.

LISTORI, G. e ESPOSTI, R. Horizontal Price Transmission in Agricultural Markets: Fundamental Concepts and Open Empirical Issues. Bio-based and Applied Economics. Vol.1, n.1, p.81 - 108, 2012.

MARGARIDO, M., SOUSA, E. Formação de Preço da Soja no Brasil. Agricultura em São Paulo. Vol. 45, n.2, p 52-61, 1998 
MARSHALL, A. Principles of Economics. 8th Edition, New York: Macmillan, 1980

MATTOS, F. L.; SILVEIRA, R. L. F. The effects of Brazilian second (winter) corn crop on price seasonality, basis behavior and integration to international market. In: NCCC134 CONFERENCE ON APPLIED COMMODITY PRICE ANALYSIS, FORECASTING AND MARKET RISK MANAGEMENT, St. Louis, Missouri, Apr. 20-21, 2015.

MEYER, J.; CRAMON-TAUBADEL, S. Asymmetric Price Transmission: A Survey. Journal of Agricultural Economics. Vol. 55, n. 3, p. 581-611, 2004

MILJKOVIC, D. The Law of One Price in International Trade: A Critical Review. Review of Agricultural Economics Vol. 21, n.1, p. 126-139, 1999

MIRANDA, R. A.; GARCIA, J. C. Avaliação econômica da safrinha de milho no Mato grosso e Paraná em 2012. Comunicado Técnico 200, Embrapa, Sete Lagoas, MG, Dezembro, 2012.

MORAES, M. C. M. M.; CHEN, R. F.F.; CARAUTA, K. C. S.; YONENAGA, W. H. Dynamic risk assessment model to the corn production system in Mato Grosso, Brazil. In: INTERNATIONAL CONFERENCE OF AGRICULTURAL EXONOMISTS, 29 ${ }^{\mathrm{TH}}$, 2015, Milan, Italy.

MORATOYA, E. E. Transmissão e volatilidade de preços das commodities agrícolas: soja e milho. 2014. 90 f. Dissertação (Mestrado em Agronegócio) Universidade Federal de Goiás - EA/ UFG, Goiânia.

MUNDLAK, Y., LARSON, D.F. On the Transmission of World Agricultural Prices. World Bank Economic Review. Vol. 6, n.3, p.399-422, 1992

PIPPENGER, J., PHILLIPS, L. Strictly Speaking, The Law of One Price Works in Commodity Markets. Working Paper, University of California, Santa Barbara, 2007

RICHARDSON, J. D. Some Empirical Evidence on Commodity Arbitrage and the Law of One Price, Journal of International Economics, Vol. 8, n. 2, p. 341-351, 1978

SAADI, H. Price Co-movements in International Markets and Their Impacts on Price Dynamics. In: Piot-Lepetit, I.; M'Barek, R. (eds.). Methods to Analyse Agricultural Commodity Price Volatility. Springer, p.149-164, 2011.

SANTOS, V. F.; PEREIRA, M. W. G.; VIEIRA, W. C. Transmissão de preços do milho entre os mercados externos e internos. In: Congresso Brasileiro de Economia e Sociologia Rural, Londrina, 2007.

SILVA, O.; FRASCAROLI, B.; MAIA, S. Transmissão de Preços no Mercado Internacional da Soja: Uma Abordagem Pelos Modelos ARMAX e VAR. In: XXXIII Encontro Nacional de Economia, Natal, 2005

SOUSA, E. P.; BRAGA, M. J.; CUNHA, D. A. Interdependência dos preços do milho no sul brasileiro. Revista de Economia, v. 36, n. 2, p. 71-90, maio/ago. 2010.

SOUSA, E.; CAMPOS, A.C. Transmissão de preços no mercado brasileiro de soja. In: XLVI Congresso Brasileiro de Economia e Sociologia Rural, Rio Branco, 2008. 
STIGLER, M. Commodity Prices: Theoretical and Empirical Properties. In: Prakash, A. (ed.), Safeguarding Food Security in Volatile Global Markets. Roma: FAO, p. 25-41, 2011

TABOSA, F.J.S.; IRFFI, G.D.; PENNA, C.M. Análise de clube de convergência para o mercado brasileiro do milho. Revista de Economia e Agronegócio, v. 11, n. 1, 2014.

TARDELLI, B.L.S. O escoamento de soja de Mato Grosso para exportação: uma análise de integração espacial de mercados e dos impactos da redução dos custos de transporte. Dissertação (Mestrado em Economia). Faculdade de Ciências Econômicas, Universidade Federal do Rio Grande do Sul, 2013

USDA, United States Department of Agrilcuture. Grain: World Markets and Trade. Publicado em: Agosto de 2015. Acesso em: http://www.usda.gov/oce/commodity/wasde/latest.pdf.

WESTERICH FILHO, V. A. Transmissão de preços no mercado de milho brasileiro: um estudo das regiões Sul e Centro-Oeste. 2014. 124 f. Dissertação (Mestrado em Agronegócios) - Universidade Federal do Rio Grande do Sul, Porto Alegre. 